\title{
A POSTERIORI ERROR ANALYSIS FOR HIGHER ORDER DISSIPATIVE METHODS FOR EVOLUTION PROBLEMS
}

\author{
CHARALAMBOS MAKRIDAKIS AND RICARDO H. NOCHETTO
}

\begin{abstract}
We prove a posteriori error estimates for time discretizations by the discontinuous Galerkin method and the corresponding implicit Runge-KuttaRadau method of arbitrary order for both linear and nonlinear evolution problems. The key ingredient is a novel higher order reconstruction $\widehat{U}$ of the discrete solution $U$, which restores continuity and leads to the differential equation $\widehat{U}^{\prime}+\Pi \mathfrak{F}(U)=F$ for a suitable interpolation operator $\Pi$. The error analysis hinges on careful energy arguments and the monotonicity of the operator $\mathfrak{F}$, in particular its angle bounded structure. We discuss applications to linear PDE such as the convection-diffusion equation and the wave equation, and nonlinear PDE corresponding to subgradient operators such as the $p$-Laplacian and minimal surfaces, as well as Lipschitz and noncoercive operators.
\end{abstract}

\section{INTRODUCTION}

Many contributions in the last few years have been devoted to a posteriori error analysis for time dependent problems. Most of the work has been done for linear or nonlinear dissipative problems by considering time discretizations based on the backward Euler method or on higher order discontinuous Galerkin methods, cf. e.g., $[9,7,8,17,27,28]$ and $[15,16,18]$.

If $u$ denotes the exact solution, $U$ its approximation and $\|\cdot\|$ a norm, we would like to obtain a posteriori error estimates of the form

$$
\|u-U\| \leq \eta(U, f)
$$

where the estimator $\eta(U, f)$ exhibits the following properties:

- $\eta(U, f)$ is a computable quantity which solely depends on the approximate solution $U$ and the data $f$ of the problem;

- $\eta(U, f)$ is of optimal order and entails minimal regularity;

- $\eta(U, f)$ utilizes explicit and easily computable constants.

Date: August 10, 2004.

2000 Mathematics Subject Classification. 65M15, 65M50.

Key words and phrases. A posteriori error analysis, discontinuous Galerkin, Runge-KuttaRadau, reconstruction, angle bounded, energy method. 
In this paper we propose an approach to this problem for the time discretization, via the discontinuous Galerkin method $\mathrm{dG}(q)$ and the corresponding implicit RungeKutta-Radau IIA method IRK-R $(q)$ of any order $q \geq 1$, of the initial value problem

$$
u^{\prime}+\mathfrak{F}(u)=f, \quad u(0)=u_{0},
$$

in a Hilbert space $(\mathcal{H},\langle\cdot, \cdot\rangle,|\cdot|)$. The operator $\mathfrak{F}: D(\mathfrak{F}) \rightarrow \mathcal{H}$ will be angle bounded

$$
\langle\mathfrak{F}(v)-\mathfrak{F}(w), w-z\rangle \leq \gamma^{2}\langle\mathfrak{F}(v)-\mathfrak{F}(z), v-z\rangle \quad \forall v, w, z \in D(\mathfrak{F})
$$

with $\gamma \geq \frac{1}{2}[5,26,27]$; this structural condition implies monotonicity but it is indeed much stronger. We will develop most of the theory under the assumption (1.2), which yields optimal order-regularity error estimates. We will also consider monotone operators for which optimal order comes at the expense of extra regularity. Our results are valid for $q \geq 1$, are based on the dissipative structure of both $\mathrm{dG}(q)$ and IRK-R $(q)$, and extend the optimal error estimates of Nochetto, Savaré and Verdi for the implicit Euler method $(q=1)$ [26, 27]; see also [23, 24]. In addition to $(1.2)$ we will assume that $\mathfrak{F}$ satisfies certain coercivity conditions for a seminorm $\|\cdot\|$ on $D(\mathfrak{F})$. In particular we will consider linear problems with smoothing effect ( $\mathfrak{F}$ is sectorial) and several nonlinear problems with dissipative character; see Sections 3, 4 and 5 for details on our assumptions.

In contrast to the approach of $[7,8,9,10,11,15,16,17,28]$, which is based on the strong stability of suitable dual problems, the key novel ingredient of our approach to a posteriori error analysis is a higher order reconstruction $\widehat{U}$, of degree $q+1$, which yields the differential equation

$$
\hat{U}^{\prime}+\Pi \mathfrak{F}(U)=F
$$

where $\widehat{U}$ is a suitable continuous interpolant of the discontinuous discrete solution $U, \Pi$ is an operator into a space of discontinuous polynomials $\mathcal{V}_{k}(q)$ of degree $\leq q$, and $F$ is an approximation of $f$ within $\mathcal{V}_{k}(q)$. Expression (1.3) extends to $q>1$ the pointwise representations of $[25,26,27]$ for $q=1$. In these works $\widehat{U}$ is the natural piecewise linear interpolant of the piecewise constant backward Euler approximation $U$. Rewriting (1.3) in the form

$$
\widehat{U}^{\prime}+\mathfrak{F}(U)=F+\mathfrak{F}(U)-\Pi \mathfrak{F}(U)=: \mathfrak{R}
$$

reveals the fundamental principle behind our a posteriori error analysis: the residual $\Re$ measures the amount by which the pair $(\widehat{U}, U)$ misses to be a solution of (1.1). Therefore, stability of the continuous problem (1.1) dictates error estimates in terms of $\mathfrak{R}$. In particular, we will study in detail the relation between our results and those derived by duality for (linear) sectorial operators [9] (see Section 3.1). Regarding $(\widehat{U}, U)$ as a relaxed solution is a natural concept developed in [25] in Banach spaces for $q=1$. Higher order reconstruction is also crucial for conservative schemes such as the Crank-Nicolson method [2]. Theories in both [2, 25] differ from that herein. 
We next recall the two time discretizations $\mathrm{dG}(q)$ and $\operatorname{IRK}-\mathrm{R}(q)$ we are interested in. Let $0=t_{0}<t_{1}<\cdots<t_{N}=T$ be a partition $\mathfrak{P}$ of $[0, T], I_{n}:=\left(t_{n}, t_{n+1}\right]$, and $k_{n}:=t_{n+1}-t_{n}$ be the variable time-step. We denote by $\mathbb{P}(q)$ the space of polynomials of degree $\leq q$, and by $\mathcal{V}_{k}(q)$ the space of discontinuous piecewise polynomials of degree $\leq q$ over $\mathfrak{P}$, both with values in $D(\mathfrak{F}): g \in \mathcal{V}_{k}(q)$ reads

$$
\left.g\right|_{I_{n}}(t)=\sum_{j=0}^{q} t^{j} w_{j} \quad\left(w_{j} \in D(\mathfrak{F}) \quad 0 \leq j \leq q\right) .
$$

The discontinuous Galerkin method $\mathrm{dG}(q)$ of order $q \geq 1$ is defined as follows [7, 8, $9,10,11,14,15,16,17,21,14,30]$ : given $U_{0}:=u_{0}$, seek $U \in \mathcal{V}_{k}(q)$ such that

$$
\int_{I_{n}}\left(\left\langle U^{\prime}, v\right\rangle+\langle\mathfrak{F}(U), v\rangle\right) d t+\left\langle U_{n}^{+}-U_{n}, v_{n}^{+}\right\rangle=\int_{I_{n}}\langle f, v\rangle d t, \quad \forall v \in \mathbb{P}(q)
$$

for $0 \leq n \leq N-1$; hereafter $v_{n}:=v\left(t_{n}\right), v_{n}^{+}:=\lim _{s \downarrow} v\left(t_{n}+s\right)$. We consider also the corresponding Galerkin method with numerical quadrature at the Radau points: given $V_{0}:=u_{0}$, find $V \in \mathcal{V}_{k}(q)$ such that

$$
\int_{I_{n}}\left(\left\langle V^{\prime}, v\right\rangle+\langle I \mathfrak{F}(V), v\rangle\right) d t+\left\langle V_{n}^{+}-V_{n}, v_{n}^{+}\right\rangle=\int_{I_{n}}\langle I f, v\rangle d t, \quad \forall v \in \mathbb{P}(q)
$$

for $0 \leq n \leq N-1$. Here $I$ is the interpolation operator onto $\mathbb{P}(q)$ at the Radau points of each $I_{n}$ (see Section 2). Then $V\left(t^{n+1}\right)$ coincides with $V^{n+1}$, the solution of the Implicit Runge-Kutta Radau IIA method with $q+1$ intermediate stages [6, 13]; we will thus refer to (1.6) as IRK-R $(q)$ for short. As in [19], writing the solution $U$ of $\mathrm{dG}(q)$ in terms of Radau polynomials will prove extremely useful. This is what establishes the connection between $\mathrm{dG}(q)$ and IRK-R $(q)$, leads to (1.6) and thus to (1.3) (see Section 2).

The paper is organized as follows. We first discuss in Section 2 the reconstruction of either $U$ or $V$ above, along with the crucial pointwise representation (1.3). In Section 3 we study linear operators $\mathfrak{F}$, for which $\Pi \mathfrak{F}=\mathfrak{F}$. We examine sectorial operators in Subsection 3.1 and apply our results to convection-diffusion problems in Subsection 3.2; the estimators are of optimal order and regularity, are extremely simple since they reduce to energy dissipation or jumps $\left\|U_{n}^{+}-U_{n}\right\|$ in the energy norm, and have absolute and explicit stability constants as in [26, 27]. Compared with [9], our estimators provide additional control of the full energy norm at all times. We analyze monotone operators in Subsection 3.3 and apply our results to the wave equation in Subsection 3.4; the estimates are of optimal order but require higher regularity, as expected for hyperbolic problems (see [16]). We deal with nonlinear angle bounded operators (1.2) in Section 4. We consider subgradient operators in Subsection 4.1, Lipschitz operators in Subsection 4.2 and noncoercive operators in Subsection 4.3; the $p$-Laplacian is a relevant example. We finally derive a conditional a posteriori error estimate in Section 5 for the minimal surface operator, for which the condition is also a posteriori and thus verifiable. 


\section{ReCOnstruction}

In this section we derive the representation formula (1.3) for both $\mathrm{dG}(q)$ and $\operatorname{IRK}-\mathrm{R}(q)$, namely,

$$
\hat{U}^{\prime}+\mathfrak{F}(U)=F+\mathfrak{F}(U)-\Pi \mathfrak{F}(U),
$$

where $F:=\Pi f, \Pi=P$ is the $L^{2}$ projection onto $\mathcal{V}_{k}(q)$ for $\operatorname{dG}(q)$ and $\Pi=I$ is the Lagrange interpolation operator at the Radau points for $\operatorname{IRK}-\mathrm{R}(q)$. These two methods are indeed closely related [19, 21].

2.1. Reconstruction Operator. Let $\left\{\tau_{j}\right\}_{j=1}^{q+1}$ be the Radau points in $[0,1]$. Then $0<\tau_{1}<\cdots<\tau_{q+1}=1$ and for appropriate weights $\left\{w_{j}\right\}_{j=1}^{q+1}$ the Radau integration rule on $[0,1]$

$$
\int_{0}^{1} g(\tau) d \tau \cong \sum_{j=1}^{q+1} w_{j} g\left(\tau_{j}\right)
$$

is exact for all polynomials of degree $\leq 2 q$. Let $\left\{\ell_{i}\right\}_{i=1}^{q+1} \subset \mathbb{P}_{q}$ and $\left\{\widehat{\ell}_{i}\right\}_{i=0}^{q+1} \subset \mathbb{P}_{q+1}$ be the Lagrange polynomials associated with either $\left\{\tau_{j}\right\}_{j=1}^{q+1}$ or $\left\{\tau_{j}\right\}_{j=0}^{q+1}$ with $\tau_{0}=0$. The corresponding Radau points in $\bar{I}_{n}$ are denoted by $t_{n, j}$, the Lagrange polynomials by $\ell_{n, i}, \widehat{\ell}_{n, i}$, and they satisfy

$$
\begin{aligned}
& t_{n, j}=t_{n}+\tau_{j} k_{n} \quad j=0, \ldots, q+1 \quad\left(t_{n, 0}=t_{n}, t_{n, q+1}=t_{n+1}\right) \\
& \ell_{n, i}(t)=\ell_{i}(\tau), \widehat{\ell}_{n, i}(t)=\widehat{\ell}_{i}(\tau), \quad t=t_{n}+\tau k_{n} .
\end{aligned}
$$

The quadrature (2.2) induces a similar formula in $I_{n}$ with nodes $\left\{t_{n, j}\right\}_{j=1}^{q+1}$ and weights $w_{n, i}=k_{n} w_{i}$. In addition, let the interpolation operator $I: C[0, T] \rightarrow \mathcal{V}_{k}(q)$ be

$$
\left.I w\right|_{I_{n}}(t):=\sum_{j=1}^{q+1} \ell_{n, j}(t) w\left(t_{n, j}\right) .
$$

Consequently, if $V$ is a polynomial in $\mathbb{P}_{q}\left(I_{n}\right)$ in $t$, then $V(t)=I V(t)$.

The reconstruction operator $\hat{I}: \mathcal{V}_{k}(q) \rightarrow \mathcal{V}_{k}(q+1)$ is now defined as follows: $\left.\widehat{U}\right|_{I_{n}}=\left.\hat{I} U\right|_{I_{n}} \in \mathbb{P}(q+1)$ satisfies

$$
\begin{aligned}
& \widehat{U}_{n}^{+}=U_{n} . \\
& \int_{I_{n}}\left\langle\widehat{U}^{\prime}, v\right\rangle d t+\int_{I_{n}}\left\langle U^{\prime}, v\right\rangle d t+\left\langle U_{n}^{+}-U_{n}, v_{n}^{+}\right\rangle=0, \quad \forall v \in \mathbb{P}(q) .
\end{aligned}
$$

In the sequel, we show that $\widehat{U}$ is well defined and exhibits some useful properties.

Lemma 2.1 (Reconstruction). $\widehat{U}$ is uniquely defined by (2.5), is globaly continuous, and satisfies

$$
\widehat{U}\left(t_{n, j}\right)=U\left(t_{n, j}\right), \quad j=0, \ldots, q+1 \quad\left(U\left(t_{n, 0}\right)=U_{n}\right) .
$$


Proof. Integrating (2.5) by parts we get

$$
\begin{aligned}
-\int_{I_{n}}\left\langle\widehat{U}, v^{\prime}\right\rangle d t+\left\langle\widehat{U}_{n+1}^{-}, v_{n+1}^{-}\right\rangle & -\left\langle\widehat{U}_{n}^{+}, v_{n}^{+}\right\rangle \\
& =-\int_{I_{n}}\left\langle U, v^{\prime}\right\rangle d t+\left\langle U_{n+1}^{-}, v_{n+1}^{-}\right\rangle-\left\langle U_{n}, v_{n}^{+}\right\rangle .
\end{aligned}
$$

Since $\widehat{U}_{n}^{+}=U_{n}$, selecting $v$ constant in time we get that $\widehat{U}_{n+1}^{-}=U_{n+1}^{-}=U_{n+1}$. Since $t_{n, q+1}=t_{n+1}$, using the exactness of the Radau integration rule (2.2) in $I_{n}$, (2.6) can be written as

$$
\sum_{i=1}^{q} w_{n, i}\left\langle\widehat{U}_{n, i}, v_{n, i}^{\prime}\right\rangle=\sum_{i=1}^{q} w_{n, i}\left\langle U_{n, i}, v_{n, i}^{\prime}\right\rangle \quad\left(w_{n, i}=k_{n} w_{i}\right) .
$$

Since $v$ is arbitrary in $\mathbb{P}(q)$, we obtain $\widehat{U}_{n, j}=U_{n, j}$ for $1 \leq j \leq q$. This completes the proof.

A consequence of the fact that $\widehat{U}$ interpolates $U$ at the Radau points is the following crucial properties for the estimates to follow.

Lemma 2.2 (Properties of $\widehat{U}$ ). The following error representation is valid

$$
\left.(\widehat{U}-U)\right|_{I_{n}}(t)=\widehat{\ell}_{n, 0}(t)\left(U_{n}-U_{n}^{+}\right), \quad \forall t \in I_{n} .
$$

If

$$
\alpha_{p}:=\left(\int_{0}^{1}\left|\widehat{\ell}_{0}(\tau)\right|^{p} d \tau\right)^{1 / p}, \quad \forall 1 \leq p \leq \infty,
$$

then for any semi-norm $\|\cdot\|$ in $\mathcal{H}$

$$
\left(\int_{I_{n}}\|\widehat{U}-U\|^{p} d t\right)^{1 / p}=\alpha_{p} k_{n}^{1 / p}\left\|U_{n}^{+}-U_{n}\right\|, \quad \forall 1 \leq p \leq \infty .
$$

Proof. Note that $\widehat{U}-U$ in $I_{n}$ is a polynomial of degree $q+1$ which, in view of Lemma 2.1, vanishes at the Radau points $\left\{t_{n, j}\right\}_{j=1}^{q+1}$. Since $(\widehat{U}-U)\left(t_{n, 0}\right)=U_{n}-U_{n}^{+}$, we readily deduce $(2.7)$. Since

$$
\int_{I_{n}}\left|\widehat{\ell}_{n, 0}(t)\right|^{p} d t=k_{n} \int_{0}^{1}\left|\widehat{\ell}_{0}(\tau)\right|^{p} d \tau=k_{n} \alpha_{p}^{p}
$$

then (2.9) follows immediately. The proof is thus complete.

2.2. Discontinuous Galerkin Methods. In view of (2.5), we can rewrite (1.5) as

$$
\int_{I_{n}}\left(\left\langle\widehat{U}^{\prime}, v\right\rangle+\langle\mathfrak{F}(U), v\rangle\right) d t=\int_{I_{n}}\langle f, v\rangle d t, \quad \forall v \in \mathbb{P}(q) .
$$

If $P$ is the piecewise $L^{2}$ projection onto $\mathcal{V}_{k}(q)$, then (2.10) readily implies

$$
\widehat{U}^{\prime}+P \mathfrak{F}(U)=F,
$$


with $F:=P f$. For piecewise constant solutions $U$, that is $q=0$, we have $P \mathfrak{F}(U)=$ $\mathfrak{F}(U)$. An expression similar to (2.11) was first used in [27] for subgradient and angle-bounded operators, and later extended in [25] to accretive operators in Banach spaces for $q=1$ and in [2] to the Crank-Nicolson method in Hilbert spaces.

2.3. Runge-Kutta-Radau Methods. We now consider the Implicit Runge-Kutta Radau IIA method $\operatorname{IRK}-\mathrm{R}(q)$ with $q+1$ intermediate stages $\left\{V_{n, j}\right\}_{j=1}^{q+1}[6,13]$. It is known that the coefficients of $\operatorname{IRK}-\mathrm{R}(q)$ are

$$
a_{i j}=\int_{0}^{\tau_{i}} \ell_{j}(\tau) d \tau, \quad b_{i}=\int_{0}^{1} \ell_{i}(\tau) d \tau\left(=a_{q+1, i}\right), \quad \forall 1 \leq i, j \leq q+1,
$$

and that the following implicit relation for $\left\{V_{n, j}\right\}_{j=1}^{q+1}$ holds

$$
V_{n, i}-V_{n}+k_{n} \sum_{j=1}^{q+1} a_{i, j}\left(\mathfrak{F}\left(V_{n, j}\right)-f_{n, j}\right)=0,
$$

where $f_{n, j}:=f\left(t_{n, j}\right)$. It is instructive to see the connection between (2.13) and (1.6), which hinges on the interpolant $\hat{V}=\hat{I} V$.

Lemma 2.3 (Equivalence between (1.6) and (2.13)). Formulations (1.6) and (2.13)

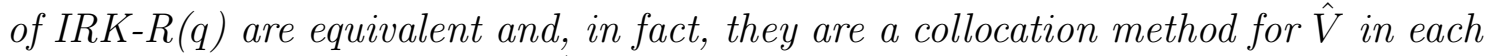
interval $I_{n}$ with starting value $\hat{V}_{n, 0}=V_{n}$, namely

$$
\hat{V}_{n, i}^{\prime}+\mathfrak{F}\left(\hat{V}_{n, i}\right)=f_{n, i}, \quad \forall 1 \leq i \leq q+1 .
$$

Proof. We first resort to the exactness of the Radau quadrature for polynomials of degree $\leq 2 q$ to realize that

$$
\langle I \mathfrak{F}(V)-I f, v\rangle=\sum_{j=1}^{q+1} w_{n, j}\left\langle\mathfrak{F}\left(V_{n, j}\right)-f_{n, j}, v\left(t_{n, j}\right)\right\rangle, \quad \forall v \in \mathbb{P}(q) .
$$

Therefore, (1.6) yields the expression

$$
\int_{I_{n}}\left\langle V^{\prime}, v\right\rangle d t+\left\langle V_{n}^{+}-V_{n}, v_{n}^{+}\right\rangle+\sum_{j=1}^{q+1} w_{n, j}\left\langle\mathfrak{F}\left(V_{n, j}\right)-f_{n, j}, v\left(t_{n, j}\right)\right\rangle=0, \quad \forall v \in \mathbb{P}(q),
$$

or, with the help of (2.5), the simpler expression

$$
\int_{I_{n}}\left\langle\hat{V}^{\prime}, v\right\rangle d t+\sum_{j=1}^{q+1} w_{n, j}\left\langle\mathfrak{F}\left(V_{n, j}\right)-f_{n, j}, v\left(t_{n, j}\right)\right\rangle=0, \quad \forall v \in \mathbb{P}(q) .
$$


Since $\hat{V}^{\prime}$ is a polynomial of degree $\leq q$, taking $v=\ell_{n, i}$ and making use again of the Radau quadrature and Lemma 2.2, we end up with (2.14). Consequently

$$
\hat{V}^{\prime}(t)=\sum_{j=1}^{q+1}\left(f_{n, j}-\mathfrak{F}\left(V_{n, j}\right)\right) \ell_{n, j}(t), \quad \forall t \in I_{n},
$$

whence

$$
\begin{aligned}
V_{n, i}-V_{n} & =\int_{t_{n}}^{t_{n, i}} \hat{V}^{\prime}(t) d t \\
& =\sum_{j=1}^{q+1}\left(f_{n, j}-\mathfrak{F}\left(V_{n, j}\right)\right) \int_{t_{n}}^{t_{n, i}} \ell_{n, j}(t) d t=k_{n} \sum_{j=1}^{q+1} a_{i, j}\left(f_{n, j}-\mathfrak{F}\left(V_{n, j}\right)\right),
\end{aligned}
$$

which is (2.13). This completes the proof.

Expression (2.17) also reads

$$
\int_{I_{n}}\left(\left\langle\hat{V}^{\prime}, v\right\rangle+\langle I \mathfrak{F}(V), v\rangle\right) d t=\int_{I_{n}}\langle I f, v\rangle d t, \quad \forall v \in \mathbb{P}(q),
$$

or equivalently

$$
\widehat{V}^{\prime}+I \mathfrak{F}(V)=F,
$$

with $F:=I f$. A comparison of (2.18) with (2.11) leads to the interesting conclusion that the pointwise representations of $\mathrm{dG}(q)$ and $\operatorname{IRK}-\mathrm{R}(q)$ differ only in the form of the operator acting on $\mathfrak{F}$ and $f$. This will be instrumental below.

\section{A Posteriori Error Estimates for Linear Operators}

In this section we assume that $\mathfrak{F}: D(\mathfrak{F}) \rightarrow \mathcal{H}$ is linear, whence $\Pi \mathfrak{F}(U)=\mathfrak{F}(U)$, and $F=\Pi f$ for either $\mathrm{dG}(q)$ or $\operatorname{IRK}-\mathrm{R}(q)$ and (2.1) becomes

$$
\hat{U}^{\prime}+\mathfrak{F}(U)=F,
$$

as in $[25,26,27]$. In view of $(3.1)$, we will now examine both methods at once but distinguish between sectorial and monotone operators.

3.1. Sectorial Operators. For $\mathfrak{F}: D(\mathfrak{F}) \rightarrow \mathcal{H}$ monotone, we define the energy semi-norm associated with $\mathfrak{F}$ by

$$
\|v\|:=\langle\mathfrak{F}(v), v\rangle^{\frac{1}{2}}, \quad \forall v \in D(\mathfrak{F}),
$$

and $\mathcal{V}:=\{v \in \mathcal{H}:\|v\|<\infty\}$. In addition, we assume that $\mathfrak{F}$ satisfies the strong sector condition

$$
|\langle\mathfrak{F}(v), w\rangle|^{2} \leq 4 \gamma^{2}\|v\|^{2}\|w\|^{2}, \quad \forall v, w \in D(\mathfrak{F}),
$$


which implies $\gamma \geq 1 / 2$; note that $\gamma=1 / 2$ in case $\mathfrak{F}$ is selfadjoint. Condition (3.3) is equivalent to the following inequality for the skew-symmetric part of the operator $[5$, Proposition 1]

$$
|\langle\mathfrak{F}(v), w\rangle-\langle\mathfrak{F}(w), v\rangle| \leq 2 \mu\|v\|\|w\|, \quad \forall v, w \in D(\mathfrak{F}),
$$

with $\gamma^{2}=\left(\mu^{2}+1\right) / 4$; note that $\mu=0$ if $\mathfrak{F}$ is symmetric. We observe that (3.3) implies that $\mathfrak{F}$ is continuous and $\|\mathfrak{F}(v)\|_{\star}=\sup _{w \in D(\mathfrak{F})} \frac{\langle\mathfrak{F}(v), w\rangle}{\|w\|}$ satisfies

$$
\frac{1}{4 \gamma^{2}}\|\mathfrak{F}(v)\|_{\star}^{2} \leq\|v\|^{2} \leq\|\mathfrak{F}(v)\|_{\star}^{2}, \quad \forall v \in D(\mathfrak{F}) .
$$

Lemma 3.1 (Linear Angle-Bounded Operators). The strong sector condition (3.3) is equivalent to the $\gamma^{2}$-angle-bounded condition

$$
\langle\mathfrak{F}(v-w), w-z\rangle \leq \gamma^{2}\langle\mathfrak{F}(v-z), v-z\rangle \quad \forall v, w, z \in D(\mathfrak{F}) .
$$

Proof. We simply set $\tilde{v}=v-z$ and $\tilde{w}=w-z$ in (3.6) to get the equivalent formulation (we omit the tildes)

$$
\langle\mathfrak{F}(v), w\rangle \leq \gamma^{2}\langle\mathfrak{F}(v), v\rangle+\langle\mathfrak{F}(w), w\rangle, \quad \forall v, w \in D(\mathfrak{F}) .
$$

Then replace $v$ by $\lambda v$ with $\lambda \in \mathbb{R}$, and argue with the resulting quadratic inequality in $\lambda$ to realize that (3.3) and (3.7) are equivalent.

Lemma 3.2 (Coercivity). If $\mathfrak{F}$ satisfies (3.3), then for all $v, w, z \in D(\mathfrak{F})$

$$
\langle\mathfrak{F}(v-w), w-z\rangle \leq 2 \gamma^{2}\langle\mathfrak{F}(v-z), v-z\rangle-\frac{1}{2} \max \left(\|v-w\|^{2},\|z-w\|^{2}\right) .
$$

Proof. Elementary calculations based on (3.5) yield

$$
\begin{aligned}
\langle\mathfrak{F}(v-w), w-z\rangle & =\langle\mathfrak{F}(v-w), w-v\rangle+\langle\mathfrak{F}(v-w), v-z\rangle \\
& \leq-\|v-w\|^{2}+2 \gamma\|v-w\|\|v-z\| \\
& \leq-\frac{1}{2}\|v-w\|^{2}+2 \gamma^{2}\|v-z\|^{2} \\
& \leq-\frac{1}{2}\|v-w\|^{2}+2 \gamma^{2}\langle\mathfrak{F}(v-z), v-z\rangle .
\end{aligned}
$$

On the other hand, a symmetric argument implies

$$
\begin{aligned}
\langle\mathfrak{F}(v-w), w-z\rangle & =\langle\mathfrak{F}(z-w), w-z\rangle+\langle\mathfrak{F}(v-z), w-z\rangle \\
& \leq-\|w-z\|^{2}+2 \gamma\|v-z\|\|w-z\| \\
& \leq-\frac{1}{2}\|w-z\|^{2}+2 \gamma^{2}\|v-z\|^{2} .
\end{aligned}
$$

Combining these two inequalities, we easily obtain (3.8). 
We are now ready to prove both upper and lower a posteriori error bounds. To this end, we first need to introduce the error concept $\mathfrak{E}$ :

$$
\mathfrak{E}^{2}:=\max \left(\max _{0 \leq t \leq T}|(u-\widehat{U})(t)|^{2}, \frac{1}{2} \int_{0}^{T}\|u-\widehat{U}\|^{2} d t, \frac{1}{2} \int_{0}^{T}\|u-U\|^{2} d t\right) .
$$

Theorem 3.1 (Upper Bound). If $u_{0} \in \mathcal{V}$, then the following estimate is valid for sectorial operators $\mathfrak{F}$ and for both $d G(q)$ and $\operatorname{IRK}-R(q)$ for any $q \geq 1$ :

$$
\mathfrak{E}^{2} \leq 4 \gamma^{2} \alpha_{2}^{2} \sum_{n=0}^{N-1} k_{n}\left\|U_{n}^{+}-U_{n}\right\|^{2}+2 \int_{0}^{T}\|f-F\|_{\star}^{2} d t .
$$

Proof. Subtract (3.1) from (1.1) to obtain the error equation

$$
\frac{d}{d t}(u-\widehat{U})+\mathfrak{F}(u-U)=f-F .
$$

We next multiply this equation by $u-\widehat{U}$ to see that

$$
\frac{1}{2} \frac{d}{d t}|u-\widehat{U}|^{2}-\langle\mathfrak{F}(U-u), u-\widehat{U}\rangle=\langle f-F, u-\widehat{U}\rangle
$$

whence, in view of (3.8), we deduce

$$
\frac{1}{2} \frac{d}{d t}|u-\widehat{U}|^{2}+\frac{1}{2} \max \left(\|u-U\|^{2},\|u-\widehat{U}\|^{2}\right) \leq 2 \gamma^{2}\|U-\widehat{U}\|^{2}+\|u-\widehat{U}\|\|f-F\|_{\star}
$$

The asserted estimate (3.10) follows from (2.9) after integration in time.

Remark 3.3 (Energy Dissipation). A striking property of (3.10) is that, except for data oscillation, the energy dissipation, or jump discontinuity, $\left\|U_{n}^{+}-U_{n}\right\|$ is what controls the error. This estimate for $\mathrm{dG}(q)$ as well as for $\operatorname{IRK}-\mathrm{R}(q)$ extends the estimates of Nochetto, Savaré and Verdi for the implicit Euler scheme $(q=1)$ to higher order $(q>1)$ without changing their structure [26, 27]. Similar estimates were obtained by Eriksson, Johnson and Larsson via duality [9].

Remark 3.4 (Stiff ODE). This theory applies to stiff ODE systems and yields a posteriori estimates which are dimension independent. The nature of these estimates is different though from those in $[11,15]$ in that our results incorporate energy terms and the estimators accumulate in time in the $L^{2}$ norm instead of the $L^{\infty}$ norm.

Remark 3.5 (Smooth Data A Priori Error Estimates). We assert that the error estimates in Theorem 3.1 are of optimal order-regularity provided the initial data and forcing term are smooth. To see this, we consider $f=0$ and recall the a priori estimate of [30, Theorem 12.1] extended for sectorial operators,

$$
\left(\int_{0}^{T}\|u-U\|^{2} d t\right)^{1 / 2} \leq C k^{q+1}\left(\int_{0}^{T}\left\|\partial_{t}^{q+1} u\right\|^{2} d t\right)^{1 / 2}
$$


where $k=\max _{n} k_{n}$ is the largest step-size. Since

$$
\begin{aligned}
k_{n}\left\|U_{n}^{+}-U_{n}\right\|^{2} & \leq 2 k_{n}\left\|U_{n}^{+}-u\left(t_{n}\right)\right\|^{2}+2 k_{n}\left\|U_{n}-u\left(t_{n}\right)\right\|^{2} \\
& \leq C \int_{t_{n}}^{t_{n+1}}\|U-I u\|^{2} d t+C \frac{k_{n}}{k_{n-1}} \int_{t_{n-1}}^{t_{n}}\|U-I u\|^{2} d t,
\end{aligned}
$$

we deduce from (3.11) that

$$
\sum_{n=0}^{N-1} k_{n}\left\|U_{n}^{+}-U_{n}\right\|^{2} \leq C k^{2(q+1)} \int_{0}^{T}\left\|\partial_{t}^{q+1} u\right\|^{2} d t,
$$

provided $k_{n} \leq C k_{n-1}$; the latter is a reasonable constraint between consecutive timesteps. Compared with the estimates of Eriksson, Johnson, and Larsson [7, 8, 9], which require the regularity $\max _{0 \leq t \leq T}\left|\partial_{t}^{q+1} u\right|$, we observe that both

$$
\max _{0 \leq t \leq T}\left|\partial_{t}^{q+1} u\right| \text { and }\left(\int_{0}^{T}\left\|\partial_{t}^{q+1} u\right\|^{2} d t\right)^{1 / 2}
$$

are bounded by the same constant depending on data. Therefore, in the linear case considered here, their control require the same regularity on the data $\left(u_{0}, f\right)$ of problem (1.1).

Remark 3.6 (Comparison with Duality). We now show the striking agreement between the stability constant $\gamma^{2}$ in Theorem 3.1 and the corresponding one of Eriksson, Johnson and Larsson [9] for analytic semigroups based on duality arguments for $\mathrm{dG}(q)$. The a posteriori error estimate shown in [9] has the form

$$
\max _{1 \leq n \leq N}\left|u\left(t_{n}\right)-U\left(t_{n}\right)\right| \leq C_{I} C_{S} L_{N} \max _{0 \leq n \leq N-1}\left(\left|U_{n}^{+}-U_{n}\right|+\max _{t \in I_{n}} k_{n}|f-P f|\right),
$$

where $C_{I}$ is an interpolation constant, $L_{N}$ grows logarithmicaly with respect to $k_{N}$ and $C_{S}$ is the stability constant of an homogeneous backward dual problem. A sharp bound for $C_{S}$ can be found by a simple energy argument [30]. For simplicity we consider the corresponding homogeneous forward problem

$$
w_{t}+\mathfrak{F}^{\star}(w)=0, \quad w(0)=w^{0},
$$

where $\mathfrak{F}^{\star}$ is the adjoint of $\mathfrak{F}$. Then $C_{S}$ is the constant of the strong stability estimate

$$
\left|\mathfrak{F}^{\star}(w(t))\right| \leq \frac{C_{S}}{t}\left|w^{0}\right|
$$

We assert that $C_{S} \approx \gamma$ for sectorial operators. To see this, we deal with $v=t w$ and the equation that it satisfies. Since $v_{t}=w+t w_{t}$, then

$$
v_{t t}+\mathfrak{F}^{\star}\left(v_{t}\right)=w_{t},
$$

whence

$$
\left\langle v_{t t}, v_{t}\right\rangle+\left\langle\mathfrak{F}^{\star}\left(v_{t}\right), v_{t}\right\rangle=\left\langle w_{t}, v_{t}\right\rangle=-\left\langle\mathfrak{F}^{\star}(w), v_{t}\right\rangle \leq 2 \gamma\|w\|\left\|v_{t}\right\| \leq \gamma^{2}\|w\|^{2}+\left\|v_{t}\right\|^{2} .
$$


Therefore, since $\left\langle\mathfrak{F}^{\star}\left(v_{t}\right), v_{t}\right\rangle=\left\|v_{t}\right\|^{2}$ and $\int_{0}^{t}\|w\|^{2} d s \leq \frac{1}{2}\left|w^{0}\right|^{2}$, we obtain

$$
\left|v_{t}\right|^{2} \leq 2 \gamma^{2} \int_{0}^{t}\|w\|^{2} d t+\left|w^{0}\right|^{2} \leq\left(\gamma^{2}+1\right)\left|w^{0}\right|^{2} .
$$

Moreover, the fact that $|w(t)| \leq\left|w^{0}\right|$ finally yields

$$
\left.t\left|\mathfrak{F}^{\star}(w)\right|=t\left|w_{t}\right| \leq|w|+\left|v_{t}\right| \leq\left(\gamma^{2}+1\right)^{1 / 2}+1\right)\left|w^{0}\right|
$$

which implies

$$
C_{S}=\left(\gamma^{2}+1\right)^{1 / 2}+1
$$

This shows that although the approach of this paper is based on simple but careful energy arguments, it gives a posteriori error bounds that for linear sectorial operators compare remarkably well with the estimates based on duality techniques [9].

Theorem 3.2 (Lower Bound). If $u_{0} \in \mathcal{V}$, then the following estimate is valid for sectorial operators $\mathfrak{F}$ and for both $d G(q)$ and $\operatorname{IRK} R(q)$ for any $q \geq 1$ :

$$
\alpha_{2}^{2} \sum_{n=0}^{N-1} k_{n}\left\|U_{n}^{+}-U_{n}\right\|^{2} \leq 4 \mathfrak{E}^{2}
$$

Proof. This is a trivial consequence of (2.9) because

$$
\begin{aligned}
\alpha_{2}^{2} \sum_{n=0}^{N-1} k_{n}\left\|U_{n}^{+}-U_{n}\right\|^{2} & =\int_{0}^{T}\|\widehat{U}-U\|^{2} d t \\
& \leq 2 \int_{0}^{T}\left(\|u-\widehat{U}\|^{2}+\|u-U\|^{2}\right) d t \leq 4 \mathfrak{E}^{2} .
\end{aligned}
$$

Remark 3.7 (Dominant Term). A simple by-product of (3.10) and the above proof is the following upper bound

$$
\max _{0 \leq t \leq T}|(u-\widehat{U})(t)|^{2} \leq 16 \gamma^{2} \max \left(\int_{0}^{T}\|u-\widehat{U}\|^{2}, \int_{0}^{T}\|u-U\|^{2}\right)+2 \int_{0}^{T}\|f-F\|_{\star}^{2} .
$$

This shows that, up to data oscillation, the energy error $L^{2}(\mathcal{V})$ controls the $L^{\infty}(\mathcal{H})$ error.

3.2. Application: Convection-Diffusion Equation. Let $\Omega$ be a bounded Lipschitz domain in $\mathbb{R}^{d}$ with any $d \geq 1$. Consider the initial boundary value problem

$$
\begin{array}{rlrl}
u_{t}-\epsilon^{2} \Delta u+b \cdot \nabla u+c u & =f, & & \text { in } \Omega \times[0, T], \\
u(\cdot, 0) & =u_{0}, & & \text { in } \Omega, \\
u & =0, & \text { on } \partial \Omega \times[0, T],
\end{array}
$$

with $\mathcal{H}:=L^{2}(\Omega)$ and norm $|\cdot|$. The coefficients $\boldsymbol{b} \in W^{1, \infty}(\Omega), c \in L^{\infty}(\Omega)$ satisfy

$$
|\boldsymbol{b}(x)| \leq b_{0}, \quad d(x):=-\frac{1}{2} \operatorname{div} \boldsymbol{b}(x)+c(x) \geq d_{0}^{2} \geq 0, \quad \text { a.e. } x \in \Omega,
$$


and $\epsilon^{2}>0$. The underlying elliptic operator $\mathfrak{F}(v):=-\epsilon^{2} \Delta v+\boldsymbol{b} \cdot \nabla v+c v$ has a domain $D(\mathfrak{F}):=\left\{v \in H_{0}^{1}(\Omega): \Delta v \in \mathcal{H}\right\}$, and induces the energy norm in $\mathcal{V}:=H_{0}^{1}(\Omega)$

$$
\|v\|^{2}=\langle\mathfrak{F}(v), v\rangle=\int_{\Omega}\left(\epsilon^{2}|\nabla v|^{2}+d|v|^{2}\right) d x \quad \forall v \in \mathcal{V}
$$

Let $p_{D}>0$ be the constant of Poincaré inequality

$$
p_{D}|w| \leq|\nabla w| \quad \forall w \in \mathcal{V} .
$$

The following result is well known [27, Lemma 5.1], but we prove it for completeness.

Lemma 3.8 (F is Sectorial). $\mathfrak{F}$ satisfies (3.3) with constant $\gamma$ given by

$$
\gamma^{2}=\frac{1}{4}\left(1+\frac{b_{0}^{2}}{\epsilon^{2}\left(d_{0}^{2}+\epsilon^{2} p_{D}^{2}\right)}\right)
$$

Proof. This proof hinges on (3.4). Since

$$
\|v\|^{2} \geq \epsilon^{2}|\nabla v|^{2}, \quad\|v\|^{2} \geq\left(\epsilon^{2} p_{D}^{2}+d_{0}^{2}\right)|v|^{2} \quad \forall v \in \mathcal{V},
$$

the skew-symmetric part of $\mathfrak{F}$ satisfies for all $v, w \in \mathcal{V}$

$$
|\langle\mathfrak{F}(v), w\rangle-\langle\mathfrak{F}(w), v\rangle| \leq b_{0}|\nabla v||w|+b_{0}\left|\nabla w\left\|v \mid \leq \frac{2 b_{0}}{\epsilon\left(\epsilon^{2} p_{D}^{2}+d_{0}^{2}\right)^{\frac{1}{2}}}\right\| v\|\| w \| .\right.
$$

Consequently $\mu=b_{0} \epsilon^{-1}\left(\epsilon^{2} p_{D}^{2}+d_{0}^{2}\right)^{-\frac{1}{2}}$ and the expression for $\gamma$ follows from $\gamma^{2}=$ $\left(1+\mu^{2}\right) / 4[5$, Proposition 1].

The following a posteriori error estimates is a simple consequence of Theorems 3.1 and 3.2 .

Corollary 3.1 (Error Estimates for Convection-Diffusion Equations). If $u_{0} \in \mathcal{V}$, then the following estimates are valid for the convection-diffusion problem (3.16) with $\gamma$ given by (3.19) and for both $d G(q)$ and $\operatorname{IRK} K-R(q)$ for any $q \geq 1$ :

$$
\frac{1}{4} \alpha_{2}^{2} \sum_{n=0}^{N-1} k_{n}\left\|U_{n}^{+}-U_{n}\right\|^{2} \leq \mathfrak{E}^{2} \leq 4 \gamma^{2} \alpha_{2}^{2} \sum_{n=0}^{N-1} k_{n}\left\|U_{n}^{+}-U_{n}\right\|^{2}+2 \int_{0}^{T}\|f-F\|_{\star}^{2} d t .
$$

3.3. Monotone Operators. We consider a linear operator $\mathfrak{F}$ such that $\langle\mathfrak{F}(v), v\rangle \geq$ 0 for all $v \in \mathcal{H}$. This assumption is insufficient to guarantee optimal a priori error estimates [29]. The same happens with the a posteriori error analysis.

Theorem 3.3 (Error Estimates for Monotone Operators). If $u_{0} \in D(\mathfrak{F})$, then

$$
\max _{0 \leq t \leq T}|u-\widehat{U}| \leq \alpha_{1} \sum_{n=0}^{N-1} k_{n}\left|\mathfrak{F}\left(U_{n}^{+}-U_{n}\right)\right|+\int_{0}^{T}|f-F| d t .
$$


Proof. We repeat the argument of Theorem 3.1 except that we can no longer exploit coercivity. Since

$$
\frac{1}{2} \frac{d}{d t}|u-\widehat{U}|^{2}+\langle\mathfrak{F}(u-\widehat{U}), u-\widehat{U}\rangle=\langle\mathfrak{F}(U-\widehat{U}), u-\widehat{U}\rangle+\langle f-F, u-\widehat{U}\rangle
$$

we deduce

$$
\frac{d}{d t}|u-\widehat{U}|^{2} \leq 2|u-\widehat{U}|(|\mathfrak{F}(U-\widehat{U})|+|f-F|)
$$

We now invoke the sharp Gronwall inequality

$$
\frac{d}{d t} a(t)^{2} \leq 2 a(t) b(t) \quad \Rightarrow \quad \max _{0 \leq t \leq T} a(t) \leq a(0)+\int_{0}^{T} b(t) d t
$$

which results by comparison with the ODE satisfied by $A^{2}(t)$, where $A(t)=a(0)+$ $\int_{0}^{t} b(s) d s$. The assertion finally follows from Lemma 2.2 .

3.4. Application: Wave Equation. Let $\Omega$ be a bounded Lipschitz domain in $\mathbb{R}^{d}$ for any $d \geq 1$. We consider the initial boundary value problem:

$$
\begin{array}{lr}
v_{t t}-\Delta v=g, & \text { in } \Omega \times(0, T), \\
v(0)=v^{0}, v_{t}(0)=v^{1}, & \text { in } \Omega, \\
\left.v\right|_{\partial \Omega}=0, & \text { on } \partial \Omega \times(0, T),
\end{array}
$$

with $D(-\Delta)=H^{2}(\Omega) \cap H_{0}^{1}(\Omega)$. To write $(3.20)$ in the form $u_{t}+\mathfrak{F}(u)=f$, we set $u=\left(u_{1}, u_{2}\right)$ and reduce the order as follows:

$$
u=\left[\begin{array}{c}
v \\
v_{t}
\end{array}\right], \quad \mathfrak{F}(u)=-\left[\begin{array}{c}
u_{2} \\
\Delta u_{1}
\end{array}\right], \quad f=\left[\begin{array}{l}
0 \\
g
\end{array}\right] .
$$

Let $U=\left(U_{1}, U_{2}\right)$ be either the $\mathrm{dG}(q)$ or $\operatorname{IRK}-\mathrm{R}(q)$ approximation of $u$, and let $G=\Pi g$. The next issue is to state the functional setting. We start with $\mathcal{H}:=$ $H_{0}^{1}(\Omega) \times L^{2}(\Omega)$ with scalar product $\langle v, w\rangle=\left\langle\nabla v_{1}, \nabla w_{1}\right\rangle+\left\langle v_{2}, w_{2}\right\rangle$ and corresponding norm $|\cdot|$; hence $\langle\mathfrak{F}(v), v\rangle=0$ for all $v \in D(\mathfrak{F})=D(-\Delta) \times L^{2}(\Omega)$. The error is then

$$
\mathfrak{E}=\max _{0 \leq t \leq T}\left(\left\|\nabla\left(u_{1}-\widehat{U}_{1}\right)\right\|_{L^{2}(\Omega)}^{2}+\left\|u_{2}-\widehat{U}_{2}\right\|_{L^{2}(\Omega)}^{2}\right)^{\frac{1}{2}} .
$$

Corollary 3.2 (Energy Norm Estimate). If $v^{0} \in D(-\Delta)$ and $v^{1} \in H_{0}^{1}(\Omega)$, then

$\mathfrak{E} \leq \alpha_{1} \sum_{n=0}^{N-1} k_{n}\left(\left\|\Delta\left(U_{1, n}^{+}-U_{1, n}\right)\right\|_{L^{2}(\Omega)}^{2}+\left\|\nabla\left(U_{2, n}^{+}-U_{2, n}\right)\right\|_{L^{2}(\Omega)}^{2}\right)^{\frac{1}{2}}+\int_{0}^{T}\|g-G\|_{L^{2}(\Omega)}$.

This estimate provides an optimal order error bound at the expense of additional regularity. In order to reduce the regularity demands, we seek an alternative choice of $\mathcal{H}$ which leads to an estimate in a weaker norm but also with lower data regularity requirements. 
Let $T:=(-\Delta)^{-1}$ be the restriction to $L^{2}(\Omega)$ of the inverse Laplacian with zero Dirichlet condition. Let $\mathcal{H}:=L^{2}(\Omega) \times H^{-1}(\Omega)$ with scalar product, [3]

$$
\langle v, w\rangle:=\left\langle v_{1}, w_{1}\right\rangle+\left\langle T v_{2}, w_{2}\right\rangle, \quad \forall v, w \in \mathcal{H} ;
$$

hence $\langle\mathfrak{F}(w), w\rangle=-\left\langle w_{2}, w_{1}\right\rangle+\left\langle T(-\Delta) w_{1}, w_{2}\right\rangle=0$ for all $w \in D(\mathfrak{F})$. The error is

$$
\mathfrak{E}=\max _{0 \leq t \leq T}\left(\left\|u_{1}-\widehat{U}_{1}\right\|_{L^{2}(\Omega)}^{2}+\left\|u_{2}-\widehat{U}_{2}\right\|_{H^{-1}(\Omega)}^{2}\right)^{\frac{1}{2}} .
$$

Corollary 3.3 (Weak Estimate). If $v^{0} \in H_{0}^{1}(\Omega)$ and $v^{1} \in L^{2}(\Omega)$, then

$\mathfrak{E} \leq \alpha_{1} \sum_{n=0}^{N-1} k_{n}\left(\left\|\nabla\left(U_{1, n}^{+}-U_{1, n}\right)\right\|_{L^{2}(\Omega)}^{2}+\left\|U_{2, n}^{+}-U_{2, n}\right\|_{L^{2}(\Omega)}^{2}\right)^{\frac{1}{2}}+\int_{0}^{T}\|g-G\|_{H^{-1}(\Omega)} d t$.

Remark 3.9 (Hyperbolic vs. Parabolic Character). Using duality arguments, a posteriori error estimates for the fully discrete discontinuous Galerkin method were proved in [16] for $q=2$. In contrast to the parabolic case, the estimators were expressed in terms of discrete-time $L^{1}$ norms. This is due to the fact that strong stability estimates of the form (3.14) are not valid for the wave equation. Our estimators in this case are also expressed in terms of discrete-time $L^{1}$ norms. Compared to the parabolic case, the increased regularity required in the estimators appears also in [16]. This what is expected for problems of non-parabolic character as the a priori results for the Schrödinger equation show [19]; see also [3].

\section{A Posteriori Error Estimates for Nonlinear Operators}

In this section we will consider the nonlinear case. The notion of linear anglebounded operators (3.6) extends naturally to nonlinear $\mathfrak{F}[5,26,27]$.

Definition 4.1 (Nonlinear Angle-Bounded Operators). The (possibly) nonlinear operator $\mathfrak{F}$ is called $\gamma^{2}$-angle-bounded if it satisfies for some $\gamma>0$

$$
\langle\mathfrak{F}(v)-\mathfrak{F}(w), w-z\rangle \leq \gamma^{2}\langle\mathfrak{F}(v)-\mathfrak{F}(z), v-z\rangle \quad \forall v, w, z \in D(\mathfrak{F}) .
$$

Angle bounded operators are monotone because taking $z=w$ we conclude that

$$
\langle\mathfrak{F}(v)-\mathfrak{F}(w), v-w\rangle \geq 0 \quad \forall v, w \in D(\mathfrak{F}) .
$$

We will derive our results under the assumption that an amount of coercivity is inherited by (4.1). To this end we introduce the nonnegative quantity for $\eta \geq \gamma$ :

$$
\sigma_{\eta}(v, w, z):=\eta^{2}\langle\mathfrak{F}(v)-\mathfrak{F}(z), v-z\rangle-\langle\mathfrak{F}(v)-\mathfrak{F}(w), w-z\rangle \quad \forall v, w, z \in D(\mathfrak{F})
$$

and assume the following coercivity condition.

Definition 4.2 ( $p$-Coercivity). Let $D(\mathfrak{F})$ be equiped with a lower semicontinuous (l.s.c) seminorm $\|\cdot\|$. The operator $\mathfrak{F}$ is called $p$-coercive if for some $p \geq 2$ and $\eta \geq \gamma$ there exists $\delta>0$, depending on $\eta$, such that

$$
\sigma_{\eta}(v, w, z) \geq \frac{\delta}{p} \max \left(\|v-w\|^{p},\|w-z\|^{p}\right), \quad \forall v, w, z \in D(\mathfrak{F}) .
$$


This notion of coercivity is a natural extension of the linear case (3.8) in Lemma 3.2 , where $p=2, \eta=\sqrt{2} \gamma$, and $\delta=1$; likewise, we set $\mathcal{V}:=\{v \in \mathcal{H}:\|v\|<\infty\}$. Note that even in the linear case we need $\eta>\gamma$ to gain the above coercivity. Examples of (4.3) are given in Subsections 4.1 and 4.2.

We are now ready to prove a posteriori error bounds in the nonlinear case similar to Theorem 3.1. In analogy with (3.9), we introduce the following error concept $\mathfrak{E}$ :

$$
\mathfrak{E}^{2}:=\max \left(\max _{0 \leq t \leq T}|(u-\widehat{U})(t)|^{2}, \frac{\delta}{p} \int_{0}^{T}\|u-\widehat{U}\|^{p} d t, \frac{\delta}{p} \int_{0}^{T}\|u-U\|^{p} d t\right) .
$$

Theorem 4.1 (Error Bound for Nonlinear Operators). Let $\mathfrak{F}$ be $\gamma^{2}$-angle bounded and $p$-coercive with respect to the seminorm $\|\cdot\|$ for $\eta \geq \gamma$. If $p^{\star}=p /(p-1)$, then the

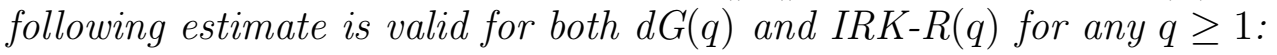

$$
\begin{aligned}
\mathfrak{E}^{2} & \leq 2 \eta^{2} \int_{0}^{T}\langle\mathfrak{F}(\widehat{U})-\mathfrak{F}(U), \widehat{U}-U\rangle d t \\
& +\frac{2}{p^{\star}}\left(\frac{4}{\delta}\right)^{p^{\star} / p} \int_{0}^{T}\left(\|\mathfrak{F}(U)-\Pi \mathfrak{F}(U)\|_{\star}^{p^{\star}}+\|f-F\|_{\star}^{p^{\star}}\right) d t .
\end{aligned}
$$

Proof. Subtract the continuous equation (1.1) from the semidiscrete counterpart (2.1), to obtain the error equation

$$
\frac{d}{d t}(\widehat{U}-u)+\mathfrak{F}(U)-\mathfrak{F}(u)=(\mathfrak{F}(U)-\Pi \mathfrak{F}(U))+(F-f) \quad \forall 0 \leq t \leq T .
$$

Testing with $\widehat{U}-u$ and using (4.3), we see that

$$
\begin{aligned}
\frac{1}{2} \frac{d}{d t}|u-\widehat{U}|^{2} & +\frac{\delta}{p} \max \left(\|u-U\|^{p},\|u-\widehat{U}\|^{p}\right) \\
& \leq \eta^{2}\langle\mathfrak{F}(U)-\mathfrak{F}(\widehat{U}), U-\widehat{U}\rangle+\langle(\mathfrak{F}(U)-\Pi \mathfrak{F}(U))+(F-f), \widehat{U}-u\rangle .
\end{aligned}
$$

Next, we use Young's inequality, $a b \leq \frac{\varepsilon}{p} a^{p}+\frac{\varepsilon^{-p^{\star} / p}}{p^{\star}} b^{p^{\star}}$, with $\varepsilon=\frac{\delta}{4}$ to arrive at

$$
\begin{aligned}
\mid\langle(\mathfrak{F}(U)-\Pi \mathfrak{F}(U)) & +(F-f), \widehat{U}-u\rangle \mid \leq \frac{\delta}{2 p}\|\widehat{U}-u\|^{p} \\
& +\frac{1}{p^{\star}}\left(\frac{\delta}{4}\right)^{-p^{\star} / p}\left(\|\mathfrak{F}(U)-\Pi \mathfrak{F}(U)\|_{\star}^{p^{\star}}+\|f-F\|_{\star}^{p^{\star}}\right),
\end{aligned}
$$

whence (4.4) follows immediately upon integration.

Remark 4.3 (Nonlinearity). In contrast with Theorem 3.1, we notice two nonlinear effects. First, we can no longer express $\langle\mathfrak{F}(\widehat{U})-\mathfrak{F}(U), \widehat{U}-U\rangle$ in terms of the jump residual $\left\|U_{n}^{+}-U_{n}\right\|^{2}$. Secondly, the new estimator $\|\mathfrak{F}(U)-\Pi \mathfrak{F}(U)\|_{\star}$ accounts for the approximation of $\mathfrak{F}(U) \notin \mathcal{V}_{k}(q)$ by piecewise polynomials of degree $\leq q$. 
4.1. Application: Subgradient Operators. A subclass of angle-bounded operators are the subgradient operators, which are characterized by the existence of a proper lower semicontinuous convex function

$$
\phi: \mathcal{H} \rightarrow(-\infty,+\infty], \quad D(\phi)=\{v \in \mathcal{H}: \phi(v)<\infty\}
$$

such that $\mathfrak{F}=\nabla \phi$ is the subgradient of $\phi$. This means that $\mathfrak{F}$ and $\phi$ satisfy

$$
\phi(w)-\phi(v)-\langle\mathfrak{F}(v), w-v\rangle \geq 0, \quad \forall v \in D(\mathfrak{F}), w \in D(\phi) .
$$

Moreover, the following well-known characterization has been used in $[26,27]$ to derive a posteriori error estimates for the implicit Euler method.

Lemma 4.4 (Subgradient Operators are 1-Angle Bounded). All subgradient operators $\mathfrak{F}$ are 1 -angle bounded. If, in addition, $\phi$ is Frechet differentiable and $\mathfrak{F}$ satisfies

$$
\|v-w\|^{p} \leq\langle\mathfrak{F}(v)-\mathfrak{F}(w), v-w\rangle, \quad \forall v, w \in D(\mathfrak{F})
$$

then $\mathfrak{F}$ is p-coercive with $\delta=1$; moreover, it holds

$$
\sigma_{1}(v, w, z) \geq \frac{1}{p}\left(\|v-w\|^{p}+\|w-z\|^{p}\right), \quad \forall v, w, z \in D(\mathfrak{F}) .
$$

Proof. Since

$\langle\mathfrak{F}(v)-\mathfrak{F}(w), w-z\rangle=\langle\mathfrak{F}(v)-\mathfrak{F}(z), v-z\rangle+\langle\mathfrak{F}(v), w-v\rangle+\langle\mathfrak{F}(w), z-w\rangle+\langle\mathfrak{F}(z), v-z\rangle$, in view of $(4.5)$, we first see that $\mathfrak{F}$ is 1 -angle bounded, i.e.

$$
\langle\mathfrak{F}(v)-\mathfrak{F}(w), w-z\rangle \leq\langle\mathfrak{F}(v)-\mathfrak{F}(z), v-z\rangle .
$$

On the other hand, using the mean value theorem in (4.5) and (4.6), we deduce

$$
\begin{aligned}
\phi(w)-\phi(v) & -\langle\mathfrak{F}(v), w-v\rangle=\int_{0}^{1}\left\langle\mathfrak{F}\left(w_{s}\right)-\mathfrak{F}(v), w_{s}-v\right\rangle \frac{d s}{s} \\
& \geq \int_{0}^{1}\left\|w_{s}-v\right\|^{p} \frac{d s}{s}=\|w-v\|^{p} \int_{0}^{1} s^{p-1} d s=\frac{1}{p}\|w-v\|^{p},
\end{aligned}
$$

where $w_{s}=s w+(1-s) v$. This implies (4.7) and concludes the proof.

In view of (4.7), we now define the error concept to be

$$
\mathfrak{E}^{2}=\max \left(\max _{0 \leq t \leq T}|(u-\widehat{U})(t)|^{2}, \frac{1}{p} \int_{0}^{T}\|u-\widehat{U}\|^{p} d t+\frac{2}{p} \int_{0}^{T}\|u-U\|^{p} d t\right) .
$$

Corollary 4.1 (Error Estimates for Subgradient Operators). Let $\mathfrak{F}=\nabla \phi$ be a subgradient operator with $\phi$ Frechet differentiable and satisfying (4.6). Then the following error estimate is valid for both $d G(q)$ and $I R K-R(q)$ for all $q \geq 1$ :

$$
\mathfrak{E}^{2} \leq 2 \int_{0}^{T}\left(\|\mathfrak{F}(\widehat{U})-\mathfrak{F}(U)\|_{\star}^{p^{\star}}+\frac{4^{p^{\star}} / p}{p^{\star}}\left(\|\mathfrak{F}(U)-\Pi \mathfrak{F}(U)\|_{\star}^{p^{\star}}+\|f-F\|_{\star}^{p^{\star}}\right)\right) d t .
$$


Proof. Since $\eta=\delta=1$ from Lemma 4.4, it suffices to show

$$
\langle\mathfrak{F}(\widehat{U})-\mathfrak{F}(U), \widehat{U}-U\rangle \leq\|\mathfrak{F}(\widehat{U})-\mathfrak{F}(U)\|_{\star}^{p^{\star}} .
$$

This follows from $\|\widehat{U}-U\|^{p} \leq\|\mathfrak{F}(\widehat{U})-\mathfrak{F}(U)\|_{\star}^{p^{\star}}$ which, in light of (4.6), results from

$$
\begin{aligned}
\|\widehat{U}-U\|^{p} & \leq\langle\mathfrak{F}(\widehat{U})-\mathfrak{F}(U), \widehat{U}-U\rangle \\
& \leq\|\mathfrak{F}(\widehat{U})-\mathfrak{F}(U)\|_{\star}\|\widehat{U}-U\| \\
& \leq \frac{1}{p^{\star}}\|\mathfrak{F}(\widehat{U})-\mathfrak{F}(U)\|_{\star}^{p^{\star}}+\frac{1}{p}\|\widehat{U}-U\|^{p} .
\end{aligned}
$$

Consequently, inserting this bound into (4.4) we obtain the asserted estimate.

Remark 4.5 ( $p$-Laplacian Operator). Given $p>2$, let $\mathfrak{F}(v):=-\operatorname{div}\left(|\nabla v|^{p-2} \nabla v\right)$ be the $p$-Laplacian operator $[4,22,27]$. This operator is the subgradient of $\phi(v):=$ $\frac{1}{p} \mid \nabla v \|_{L^{p}(\Omega)}^{p}$ in $\mathcal{H}=L^{2}(\Omega)$, and has the following coercivity property [27]

$$
\langle\mathfrak{F}(u)-\mathfrak{F}(v), u-v\rangle \geq \Lambda_{p}\|\nabla(u-v)\|^{p},
$$

for a suitable constant $\Lambda_{p}>0$. Hence $\mathfrak{F}$ is $p$-coercive with respect to the norm $\|v\|=$ $\Lambda_{p}^{1 / p}\|\nabla v\|_{L^{p}(\Omega)}$, and Corollary 4.1 applies with dual norm $\|v\|_{\star}:=\Lambda_{p}^{-1 / p}\|v\|_{W_{p^{\star}}^{-1}(\Omega)}$.

Remark 4.6 (Porous Medium Operator). Given $p>0$, let $\mathfrak{F}(v):=-\Delta\left(|v|^{p-2} v\right)$ be the porous medium operator. This prototype of degenerate operator is the subgradient of $\phi(v):=\frac{1}{p}\|v\|_{L^{p}(\Omega)}$ in $\mathcal{H}=H^{-1}(\Omega)$, and is $p$-coercive in $L^{p}(\Omega)$ [27], i.e.

$$
\langle\mathfrak{F}(v)-\mathfrak{F}(w), v-w\rangle \geq \lambda_{p}\|v-w\|_{L^{p}(\Omega)}^{p},
$$

for a suitable constant $\lambda_{p}>0$. Corollary 4.1 applies again.

4.2. Application: Lipschitz Operators. We will consider now a subclass of nonlinear operators which extend the class of linear sectorial operators of $\S 3.1$. We assume that $\mathfrak{F}$ satisfies (4.6) with $p=2$, namely,

$$
\|v-w\|^{2} \leq\langle\mathfrak{F}(v)-\mathfrak{F}(w), v-w\rangle \quad \forall v, w \in D(\mathfrak{F}),
$$

as well as the following Lipschitz condition for some $\gamma>0$

$$
\|\mathfrak{F}(v)-\mathfrak{F}(w)\|_{\star} \leq 2 \gamma\|v-w\| \quad \forall v, w \in D(\mathfrak{F})
$$

compare with (3.3). The following lemma extends Lemma 3.2, and is proved in [27, Lemma 4.3]. We present its proof here for completeness.

Lemma 4.7 (Angle-Boundedness and Coercivity). If $\mathfrak{F}$ satisfies (4.9) and (4.10), then for all $v, w, z \in D(\mathfrak{F})$

$$
\langle\mathfrak{F}(v)-\mathfrak{F}(w), w-z\rangle \leq 2 \gamma^{2}\|v-z\|^{2}-\frac{1}{2} \max \left(\|v-w\|^{2},\|z-w\|^{2}\right) .
$$


Proof. Proceeding as in Lemma 3.2, we obtain

$$
\begin{aligned}
&\langle\mathfrak{F}(v)-\mathfrak{F}(w),w-z\rangle=\langle\mathfrak{F}(v)-\mathfrak{F}(w), w-v\rangle+\langle\mathfrak{F}(v)-\mathfrak{F}(w), v-z\rangle \\
& \leq-\|v-w\|^{2}+2 \gamma\|v-w\|\|v-z\| \leq-\frac{1}{2}\|v-w\|^{2}+2 \gamma^{2}\|v-z\|^{2},
\end{aligned}
$$

as well as

$$
\begin{aligned}
&\langle\mathfrak{F}(v)-\mathfrak{F}(w),w-z\rangle=\langle\mathfrak{F}(z)-\mathfrak{F}(w), w-z\rangle+\langle\mathfrak{F}(v)-\mathfrak{F}(z), w-z\rangle \\
& \leq-\|z-w\|^{2}+2 \gamma\|v-z\|\|w-z\| \leq-\frac{1}{2}\|w-z\|^{2}+2 \gamma^{2}\|v-z\|^{2} .
\end{aligned}
$$

Combining these inequalities, we deduce the estimate (4.11).

Note that (4.11) implies (4.3) with $p=2, \eta=\sqrt{2} \gamma, \delta=1$. Therefore Theorem 4.1 is applicable with an error concept $\mathfrak{E}$ of the form:

$$
\mathfrak{E}^{2}:=\max \left(\max _{0 \leq t \leq T}|(u-\widehat{U})(t)|^{2}, \frac{1}{2} \int_{0}^{T}\|u-\widehat{U}\|^{2} d t, \frac{1}{2} \int_{0}^{T}\|u-U\|^{2} d t\right) .
$$

Corollary 4.2 (Error Estimates for Lipschitz Operators). If $u_{0} \in \mathcal{V}$ and (4.9) and (4.10) hold, then the following lower and upper bounds are valid for both $d G(q)$ and $\operatorname{IRK}-R(q)$ for all $q \geq 1$ :

$$
\begin{aligned}
\frac{1}{4} \alpha_{2}^{2} \sum_{n=0}^{N-1} k_{n}\left\|U_{n}^{+}-U_{n}\right\|^{2} \leq \mathfrak{E}^{2} & \leq 4 \gamma^{2} \alpha_{2}^{2} \sum_{n=0}^{N-1} k_{n}\left\|U_{n}^{+}-U_{n}\right\|^{2} \\
& +4 \int_{0}^{T}\left(\|\mathfrak{F}(U)-\Pi \mathfrak{F}(U)\|_{\star}^{2}+\|f-F\|_{\star}^{2}\right) d t .
\end{aligned}
$$

Proof. We note that (4.11) with $\eta=\sqrt{2} \gamma$ yields the upper bound. To derive the lower bound, we proceed as in Theorem 3.2, whose proof does not rely on the structure of $\mathfrak{F}$.

4.3. Noncoercive Operators. A class of operators that satisfy (4.9) and (4.10) can be defined as follows [1]: let $\mathfrak{F}(v):=\mathfrak{A} v-\mathfrak{B}(t, v)$, where $\mathfrak{A}$ is a positive definite, selfadjoint, linear operator on a Hilbert space $\mathcal{H}$ with domain $D(\mathfrak{A})$ dense in $\mathcal{H}$, and $\mathfrak{B}(t, \cdot): D(\mathfrak{A}) \rightarrow \mathcal{H}$ is a (possibly) nonlinear operator for all $t \in[0, T]$. We let $\mathcal{V}:=D\left(\mathfrak{A}^{1 / 2}\right)$, with norm $\|v\|:=\left|\mathfrak{A}^{1 / 2} v\right|=\langle\mathfrak{A} v, v\rangle^{1 / 2}$, and assume that $\|\cdot\|$ dominates $|\cdot|$ in $\mathcal{V}$. If we identify $\mathcal{H}$ with its dual, then the Hilbert triplet satisfies $\mathcal{V} \subset \mathcal{H} \subset \mathcal{V}^{\star}$, with $\mathcal{V}^{\star}$ the dual of $\mathcal{V}$. A natural condition for $u^{\prime}+\mathfrak{F}(u)=f$ to be of parabolic type is the one-sided Lipschitz condition on $\mathfrak{B}(t, \cdot)$,

$$
\langle\mathfrak{B}(t, v)-\mathfrak{B}(t, w), v-w\rangle \leq \lambda\|v-w\|^{2}+\mu|v-w|^{2}
$$

with a constant $0 \leq \lambda<1$; (4.13) is equivalent to the Gårding-type inequality

$$
\langle\mathfrak{F}(v)-\mathfrak{F}(w), v-w\rangle \geq(1-\lambda)\|v-w\|^{2}-\mu|v-w|^{2} .
$$


If $\mu=0,(4.14)$ reduces to (4.9). We also require the additional Lipschitz condition

$$
\|\mathfrak{B}(t, v)-\mathfrak{B}(t, w)\|_{\star} \leq L\|v-w\|
$$

with constant $L>0$. Hence, (4.10) is satisfied with $2 \gamma=L+1$. Instead of extending Corollary 4.2 to $\mu>0$, we take advantage of the structure of $\mathfrak{F}$ in the following result which is formulated in terms of the error concept

$$
\mathfrak{E}^{2}:=\max \left\{\max _{0 \leq t \leq T} e^{-3 \mu t}|(u-\widehat{U})(t)|^{2}, \frac{1-\lambda}{4} \int_{0}^{T} e^{-3 \mu t}\left(\|u-\widehat{U}\|^{2}+2\|u-U\|^{2}\right) d t\right\} .
$$

Corollary 4.3 (Error Estimate for Noncoercive Operators). If $\mathfrak{F}=\mathfrak{A}-\mathfrak{B}$ satisfies (4.13) and (4.15), and $\Gamma:=1+\frac{L^{2}}{1-\lambda}, \Lambda:=\frac{4}{1-\lambda}$, then the following error estimates are valid for both $d G(q)$ and $\operatorname{IRK} K(R)$ for all $q \geq 1$

$$
\mathfrak{E}^{2} \leq \alpha_{2}^{2} \sum_{n=0}^{N-1} k_{n} e^{-3 \mu t_{n}}\left(\Gamma\left\|U_{n}^{+}-U_{n}\right\|^{2}+2 \mu\left|U_{n}^{+}-U_{n}\right|^{2}\right)+\Lambda \int_{0}^{T} e^{-3 \mu t}\|F-f\|_{\star}^{2} d t .
$$

Proof. We first write the error equation in the form

$$
\frac{d}{d t}|\widehat{U}-u|^{2}+2\langle\mathfrak{A}(U-u), \widehat{U}-u\rangle=2\langle\mathfrak{B}(U)-\mathfrak{B}(u), \widehat{U}-u\rangle+2\langle F-f, \widehat{U}-u\rangle,
$$

and observe the elementary equality

$$
2\langle\mathfrak{A}(U-u), \widehat{U}-u\rangle=\|U-u\|^{2}+\|\widehat{U}-u\|^{2}-\|\widehat{U}-U\|^{2} .
$$

We next see that (4.13) and (4.15) imply

$$
\begin{aligned}
2\langle\mathfrak{B}(U)-\mathfrak{B}(u), \widehat{U}-u\rangle & =\langle\mathfrak{B}(U)-\mathfrak{B}(u), U-u\rangle+\langle\mathfrak{B}(\widehat{U})-\mathfrak{B}(u), \widehat{U}-u\rangle \\
& +\langle\mathfrak{B}(U)-\mathfrak{B}(u), \widehat{U}-U\rangle+\langle\mathfrak{B}(\widehat{U})-\mathfrak{B}(U), \widehat{U}-u\rangle \\
& \leq \lambda\|U-u\|^{2}+\lambda\|\widehat{U}-u\|^{2}+\mu|U-u|^{2}+\mu|\widehat{U}-u|^{2} \\
& +L\|U-u\|\|\widehat{U}-U\|+L\|\widehat{U}-u\|\|\widehat{U}-U\| .
\end{aligned}
$$

Inserting this back into the error equation, we thus arrive at

$$
\begin{aligned}
\frac{d}{d t}|\widehat{U}-u|^{2}+\frac{1-\lambda}{4}\left(2\|U-u\|^{2}\right. & \left.+\|\widehat{U}-u\|^{2}\right) \leq 3 \mu|\widehat{U}-u|^{2} \\
& +\Gamma\|\widehat{U}-U\|^{2}+2 \mu|\widehat{U}-U|^{2}+\Lambda\|F-f\|_{\star}^{2} .
\end{aligned}
$$

The asserted estimate follows easily from the Gronwall's lemma and Lemma 2.2.

\section{Conditional Estimates: Minimal Surface Equation}

For quasilinear operators $\mathfrak{F}$ the theory of $\S 4$ does not always apply. It may, in particular, be difficult to find a suitable Sobolev setting. This is the case of the minimal surface operator over a domain $\Omega$ of $\mathbb{R}^{d}$

$$
\mathfrak{F}(u):=\operatorname{div} \frac{\nabla u}{Q(u)}, \quad Q(u):=\sqrt{1+|\nabla u|^{2}},
$$


which is better studied in terms of geometric quantities. One such quantity is

$$
N(u)=\frac{(\nabla u, 1)}{Q(u)}
$$

the unit normal to the graph $\Gamma_{u}:=\{(x, u(x)): x \in \Omega\}$. The following geometrictype coercivity was first observed by Fierro and Veeser [12]:

$$
\langle\mathfrak{F}(u)-\mathfrak{F}(v), u-v\rangle=\left\langle\frac{\nabla u}{Q(u)}-\frac{\nabla v}{Q(v)}, \nabla(u-v)\right\rangle=\int_{\Omega}|N(u)-N(v)|^{2} \frac{Q(u)+Q(v)}{2} .
$$

This can be interpreted as an $L^{2}$ estimate for the normals to the graphs $\Gamma_{u}, \Gamma_{v}$ measured on them. For this to make sense we need $u, v \in W_{\infty}^{1}(\Omega)$.

Let $U$ be either the $\mathrm{dG}(q)$ or $\operatorname{IRK}-\mathrm{R}(q)$ approximation to the evolution prescribed mean curvature equation:

$$
\partial_{t} u-\operatorname{div} \frac{\nabla u}{Q(u)}=f \quad \text { in } \Omega \times(0, T),
$$

subject to an initial and lateral boundary condition $u=u_{0}$. We assume that (5.3) admits a smooth solution in $W_{\infty}^{1}(\Omega \times(0, T))$. We denote by

$$
\mathfrak{J}(U):=U_{n}^{+}-U_{n}, \quad \mathfrak{N}(U):=\frac{\nabla U}{Q(U)}-\Pi \frac{\nabla U}{Q(U)} ;
$$

the piecewise constant jump $\mathfrak{J}(U)$ is a measure of numerical dissipation and $Q(U)$ is a measure of the nonlinearity of $\mathfrak{F}$. The latter will be used next to quantify proximity to the exact solution $u$. We first set $\mathcal{H}=L^{2}(\Omega)$, and define the concept of coercivity

$$
\rho(v, w ; z):=\int_{\Omega}|N(v)-N(w)|^{2} Q(z) d x,
$$

along with that of error for $0 \leq \lambda \leq 1$ :

$$
\mathfrak{E}:=\max \left\{\max _{0 \leq t \leq T}|u-\widehat{U}|^{2} ; \int_{0}^{T}\left(\frac{1}{4} \rho(U, u ; U)+(1-\lambda) \rho(U, u ; u)\right) d t\right\}^{\frac{1}{2}} .
$$

Proposition 5.1 (Conditional Estimate). If the solution $U$ of $d G(q)$ or $\operatorname{IRK} K-R(q)$ with $q \geq 1$ satisfies the a priori condition

$$
\lambda=2\left\|\mathfrak{N}(U) Q(U)^{2}\right\|_{L^{\infty}(\Omega)} \leq 1,
$$

and $\alpha_{\infty}$ is defined as in (2.8), then

$\mathfrak{E} \leq\left\{5 \alpha_{\infty}^{2} \int_{0}^{T} \int_{\Omega} \frac{|\nabla \mathfrak{J}(U)|^{2}}{Q(U)} d x d t+9 \int_{0}^{T} \int_{\Omega}|\mathfrak{N}(U)|^{2} Q(U)^{3} d x d t\right\}^{\frac{1}{2}}+\int_{0}^{T}|F-f| d t$

Proof. Subtracting (1.1) from (2.1). we obtain the error equation

$$
\left\langle(\widehat{U}-u)^{\prime}, v\right\rangle+\langle\mathfrak{F}(U)-\mathfrak{F}(u), v\rangle=\langle\mathfrak{F}(U)-\Pi \mathfrak{F}(U), v\rangle+\langle F-f, v\rangle .
$$


We now choose $v=\widehat{U}-u$ which, in view of (2.7), reads $v=U-u+\ell \mathfrak{J}(U)$ where $\ell(t)=\ell_{n, 0}(t)$ for $t \in I_{n}$. Since

$$
\langle\mathfrak{F}(U)-\mathfrak{F}(u), U-u\rangle=\frac{1}{2}(\rho(U, u ; u)+\rho(U, u ; U)),
$$

we have

$$
\begin{aligned}
\frac{d}{d t}|u-\widehat{U}|^{2}+\rho(U, u ; u) & +\rho(U, u ; U) \leq 2|\ell||\langle\mathfrak{F}(U)-\mathfrak{F}(u), \mathfrak{J}(U)\rangle| \\
& +2\langle\mathfrak{N}(U), \nabla(\widehat{U}-u)\rangle+2\langle F-f, \widehat{U}-u)\rangle
\end{aligned}
$$

Then, since $\left|\frac{\nabla u}{Q(u)}-\frac{\nabla U}{Q(U)}\right| \leq|N(u)-N(U)|$, it is easily seen that

$$
2|\ell||\langle\mathfrak{F}(U)-\mathfrak{F}(u), \mathfrak{J}(U)\rangle| \leq \frac{1}{4} \rho(U, u ; U)+4 \alpha_{\infty}^{2} \int_{\Omega} \frac{|\nabla \mathfrak{J}(U)|^{2}}{Q(U)} d x
$$

It remains to estimate the last two terms in the right hand side of (5.6). Both terms require finding a bound for $|\nabla(U-u)|$ in terms of geometric quantities we have control of. To this end, we proceed as in [12]. If we set

$$
\mathbf{p}=(\nabla u, 1) \quad \text { and } \quad \mathbf{q}=(\nabla U, 1)
$$

then

$$
\begin{aligned}
|\mathbf{p}-\mathbf{q}| & =|| \mathbf{p}\left|\frac{\mathbf{p}}{|\mathbf{p}|}-\right| \mathbf{q}\left|\frac{\mathbf{q}}{|\mathbf{q}|}\right| \\
& \leq\left|\frac{\mathbf{p}}{|\mathbf{p}|}-\frac{\mathbf{q}}{|\mathbf{q}|}\right||\mathbf{q}|+|| \mathbf{p}\left|\frac{\mathbf{p}}{|\mathbf{p}|}-\right| \mathbf{q}\left|\frac{\mathbf{p}}{|\mathbf{p}|}\right|=\left|\frac{\mathbf{p}}{|\mathbf{p}|}-\frac{\mathbf{q}}{|\mathbf{q}|}\right||\mathbf{q}|+|| \mathbf{p}|-| \mathbf{q}||,
\end{aligned}
$$

whence

$$
|\nabla(U-u)| \leq|N(u)-N(U)| Q(U)+|Q(u)-Q(U)|
$$

In addition, for $a, b \in \mathbb{R}$, we have

$$
\frac{|b-a|}{b^{2}} \leq \frac{|b-a|}{a b}+\frac{|b-a|}{b}\left|\frac{1}{a}-\frac{1}{b}\right|=\left|\frac{1}{a}-\frac{1}{b}\right|+\left|\frac{1}{a}-\frac{1}{b}\right|^{2} a .
$$

Hence, taking $a=Q(u), b=Q(U)$ and using $\left|\frac{1}{a}-\frac{1}{b}\right| \leq|N(u)-N(U)|$, we get

$$
|Q(u)-Q(U)| \leq Q(U)^{2}|N(u)-N(U)|+Q(u) Q(U)^{2}|N(u)-N(U)|^{2} .
$$

Combining (5.7) and (5.8), and making use of $Q(U) \geq 1$, we arrive at

$$
|\nabla(U-u)| \leq 2|N(u)-N(U)| Q(U)^{2}+|N(u)-N(U)|^{2} Q(u) Q(U)^{2} .
$$

Consequently, if $\lambda=2\left\|\mathfrak{N}(U) Q(U)^{2}\right\|_{L^{\infty}(\Omega)}$, then we get

$$
|\langle\mathfrak{N}(U), \nabla(U-u)\rangle| \leq \frac{1}{4} \rho(U, u ; U)+4 \int_{\Omega} \mathfrak{N}(U)^{2} Q(U)^{3} d x+\frac{\lambda}{2} \rho(U, u ; u),
$$


whence, using $Q(U) \geq 1$,

$$
\begin{aligned}
2|\langle\mathfrak{N}(U), \nabla \widehat{U}-\nabla u\rangle| & \leq \frac{1}{2} \rho(U, u ; U)+\lambda \rho(U, u ; u) \\
& +9 \int_{\Omega}|\mathfrak{N}(U)|^{2} Q(U)^{3}+\alpha_{\infty}^{2} \int_{\Omega} \frac{|\mathfrak{J}(U)|^{2}}{Q(U)} .
\end{aligned}
$$

Inserting the above estimates back into (5.6), we find

$$
\begin{aligned}
\frac{d}{d t}|u-\widehat{U}|^{2} & +(1-\lambda) \rho(U, u ; u)+\frac{1}{4} \rho(U, u ; U) \\
& \leq 9 \int_{\Omega}|\mathfrak{N}(U)|^{2} Q(U)^{3}+5 \alpha_{\infty}^{2} \int_{\Omega} \frac{|\mathfrak{J}(U)|^{2}}{Q(U)}+2|F-f||\widehat{U}-u| .
\end{aligned}
$$

Since $\lambda \leq 1$ in view of (5.5), we thus have an expression of the form

$$
\frac{d}{d t} a^{2}(t)+b^{2}(t) \leq c^{2}(t)+2 d(t) a(t)
$$

The asserted estimate is finally a consequence of the following Gronwall-like inequality [27, Lemma 3.7]:

$$
\max \left\{\max _{0 \leq t \leq T} a(t),\left(\int_{0}^{T} b^{2}(t) d t\right)^{\frac{1}{2}}\right\} \leq\left(\int_{0}^{T} c^{2}(t) d t\right)^{\frac{1}{2}}+\int_{0}^{T} d(t) d t .
$$

Remark 5.1 (Coercivity). It is worth noticing that the second term in the definition of $\mathfrak{E}$ is not a norm but a geometric quantity without homogeneity. Therefore, the technique of Lemma 4.4 does not apply to yield coercivity. However, the minimal surface operator is 1-angle bounded from Lemma 4.4, which leads to an optimal a posteriori error estimate for polynomial degree $q=1$ as in [27]. For $q>1$ we need control of the nonlinear term $\mathfrak{N}(U)$, which vanishes otherwise, and this is achieved via the coercivity term.

Remark 5.2 (Conditional Estimates). We point out that the condition (5.5) is a posteriori, and thus verifiable. It is conceivable that, for a sufficiently fine partition of $[0, T],(5.5)$ would be valid. Conditional estimates are somehow natural for nonlinear equations but rather unusual in a posteriori error analysis. We refer to Fierro and Veeser [12] for elliptic problems of prescribed mean curvature and Lakkis and Nochetto [20] for the mean curvature flow of graphs, both for $q=1$.

\section{REFERENCES}

[1] G. Akrivis and Ch. G. Makridakis. Galerkin time stepping methods for nonlinear parabolic equations. Math. Mod. Numer. Anal., 38:261-289, 2004.

[2] G. Akrivis, Ch. G. Makridakis, and R.H. Nochetto. A posteriori error estimates for the CrankNicolson method for parabolic equations. Submitted.

[3] G. A. Baker and J. H. Bramble. Semidiscrete and single step fully discrete approximations for second order hyperbolic equations. RAIRO Anal. Numér., 13(2):75-100, 1979.

[4] H. Brézis. Problèmes unilatéraux. J. Math. Pures Appl. (9), 51:1-168, 1972. 
[5] H. Brézis and F. E. Browder. Nonlinear integral equations and systems of Hammerstein type. Advances in Math., 18(2):115-147, 1975.

[6] K. Dekker and J. G. Verwer. Stability of Runge-Kutta methods for stiff nonlinear differential equations. North-Holland Publishing Co., Amsterdam, 1984.

[7] K. Eriksson and C. Johnson. Adaptive finite element methods for parabolic problems. I. A linear model problem. SIAM J. Numer. Anal., 28(1):43-77, 1991.

[8] K. Eriksson and C. Johnson. Adaptive finite element methods for parabolic problems. IV. Nonlinear problems. SIAM J. Numer. Anal., 32(6):1729-1749, 1995.

[9] K. Eriksson, C. Johnson, and S. Larsson. Adaptive finite element methods for parabolic problems. VI. Analytic semigroups. SIAM J. Numer. Anal., 35(4):1315-1325 (electronic), 1998.

[10] K. Eriksson, C. Johnson, and V. Thomée. Time discretization of parabolic problems by the discontinuous Galerkin method. RAIRO Modél. Math. Anal. Numér., 19(4):611-643, 1985.

[11] D. Estep. A posteriori error bounds and global error control for approximation of ordinary differential equations. SIAM J. Numer. Anal., 32(1):1-48, 1995.

[12] Fierro F. and Veeser A. On the a posteriori error analysis for equations of prescribed mean curvature. Math. Comp., 72:1611-1634, 2003.

[13] E. Hairer and G. Wanner. Solving ordinary differential equations. II. Springer-Verlag, Berlin, 1991. Stiff and differential-algebraic problems.

[14] P. Jamet. Galerkin-type approximations which are discontinuous in time for parabolic equations in a variable domain. SIAM J. Numer. Anal., 15(5):912-928, 1978.

[15] C. Johnson. Error estimates and adaptive time-step control for a class of one-step methods for stiff ordinary differential equations. SIAM J. Numer. Anal., 25(4):908-926, 1988.

[16] C. Johnson. Discontinuous Galerkin finite element methods for second order hyperbolic problems. Comput. Methods Appl. Mech. Engrg., 107(1-2):117-129, 1993.

[17] C. Johnson, Y. Y. Nie, and V. Thomée. An a posteriori error estimate and adaptive timestep control for a backward Euler discretization of a parabolic problem. SIAM J. Numer. Anal., 27(2):277-291, 1990.

[18] C. Johnson and A. Szepessy. Adaptive finite element methods for conservation laws based on a posteriori error estimates. Comm. Pure Appl. Math., 48(3):199-234, 1995.

[19] O. Karakashian and Ch. Makridakis. A space-time finite element method for the nonlinear Schrödinger equation: the discontinuous Galerkin method. Math. Comp., 67(222):479-499, 1998.

[20] O. Lakkis and R. H. Nochetto. A posteriori error analysis for the mean curvature flow of graphs. SIAM J. Numer. Anal., 2004.

[21] P. Lasaint and P.-A. Raviart. On a finite element method for solving the neutron transport equation, in mathematical aspects of finite elements in partial differential equations. Proc. Sympos., Math. Res. Center, Univ. Wisconsin, Madison, Academic Press, pages 89-123. Publication No. 33, 1974.

[22] J.-L. Lions. Quelques Méthodes de Résolution des Problèmes aux Limites Non Linéaires. Dunod, 1969.

[23] G. Lippold. Error estimates for the implicit Euler approximation of an evolution inequality. Nonlinear Anal., 15(11):1077-1089, 1990.

[24] G. Lippold. Error estimates and step-size control for the approximate solution of a first order evolution equation. RAIRO Modél. Math. Anal. Numér., 25(1):111-128, 1991.

[25] R. H. Nochetto and G. Savaré. Nonlinear evolution equations governed by accretive operators in banach spaces: error control and applications. submitted.

[26] R. H. Nochetto, G Savaré, and C. Verdi. Error control for nonlinear evolution equations. C.R. Acad. Sci. Paris, Serie I, 326:1437-1442, 1998. 
[27] R. H. Nochetto, G. Savaré, and C. Verdi. A posteriori error estimates for variable time-step discretizations of nonlinear evolution equations. Comm. Pure Appl. Math., 53(5):525-589, 2000.

[28] R. H. Nochetto, A. Schmidt, and C. Verdi. A posteriori error estimation and adaptivity for degenerate parabolic problems. Math. Comp., 69(229):1-24, 2000.

[29] J. Rulla. Error analysis for implicit approximations to solutions to cauchy problems. SIAM J. Numer. Anal., 33(1):68-87, 1996.

[30] V. Thomée. Galerkin Finite Element Methods for Parabolic Problems. Springer-Verlag, Berlin, 1997.

Department of Applied Mathematics, University of Crete, 71409 Heraklion-Crete, Greece and Institute of Applied and Computational Mathematics, FORTH, 71110 Heraklion-Crete, Greece. Partially supported by the European Union RTN-network HYKe, HPRN-CT-2002-00282, and the EU Marie Curie Development Host Site, HPMD-CT-2001-00121.

URL: http://www.tem.uoc.gr/ makr

E-mail address: makr@tem.uoc.gr

Department of Mathematics and Institute for Physical Science and Technology, University of Maryland, College Park, MD 20742, USA. Partially supported by NSF Grants DMS-9971450 and DMS-0204670 and the General Research BoARd of THE UNIVERSITY OF MARYLAND.

URL: http://www.math.umd.edu/ ${ }^{\mathrm{rhh}}$

E-mail address: rhn@math.umd.edu 